$\mathrm{KN}-13$

\section{Taking on the next structural challenges posed by polycrystalline materials}

Lynne McCusker ${ }^{1}$, Christian Baerlocher ${ }^{1}$

${ }^{1}$, ETH Zurich, Zurich, Switzerland

email: mccusker@mat.ethz.ch

Three decades ago, the determination of the crystal structure of a polycrystalline material using X-ray powder diffraction (XPD) data was a challenge, requiring a dedicated and patient crystallographer. Today, the necessary tools have been developed, and such analyses are conducted worldwide in all areas of science on a routine basis. During this time, we have also watched the definition of a single crystal change. The $100 \mathrm{~mm}$ crystal previously required for data collection in a laboratory using a point detector could be reduced to $30 \mathrm{~mm}$ with an area detector, and to just a few $\mathrm{mm}$ with synchrotron microdiffraction techniques. More recently electron diffraction and X-ray Free Electron Lasers have reduced this requirement still further into the nanometer range. Does this mean that the job is done and we should seek other challenges?

Like all areas of science, it is always possible to dig deeper. It can be argued that developing methodology and instrumentation to address the initial phase problem was just the first step in the elucidation of the structure of a polycrystalline material. The structural details that become evident during the course of a Rietveld refinement continue to pose new challenges. Probably the most common of these is the presence of some element of disorder. Even with single-crystal data, the full analysis of the diffuse scattering that accompanies any disordered structure still lies within the realm of the experts, but with a little persistence some useful information can be extracted from the Bragg peaks alone. How far can we go with XPD data? For example, can the disordered organic structure-directing agents used in zeolite syntheses be located in the final product using XPD data? Yes, they can.

Can other techniques help us overcome some of the limitations inherent to XPD data? In our own research we have come to appreciate the complementarity of XPD, electron microscopy/diffraction and solid state NMR techniques. For example, the conformer disorder in D-ribose was first indicated by MAS NMR, the framework disorder in the niobium silicate AM-11 could be seen in electron microscopy images, and the unexpected structure of the inner surface of the disordered zeolite catalyst SSZ-70 could only be fully characterized via sophisticated NMR techniques. Combining techniques requires manual intervention and tenacity, but the results are worth the effort. A few examples of such studies will be presented.References:

Keywords: powder diffraction, disorder, zeolites
$\mathrm{KN}-14$

\section{New insights into making and breaking crystals}

Aurora Cruz-Cabeza ${ }^{1}$

1. School of Chemical Engineering and Analytical Sciences, The University of Manchester, Manchester, United Kingdom

email: aurora.cruzcabeza@manchester.ac.uk

At the crystallisation group at Manchester, we have had a long-standing interest in the study of crystal nucleation and growth (making crystals). Understanding, designing and controlling robust crystallisation processes is key for the production of crystalline materials. More recently, we have also developed an interest in understanding the processes of milling (breaking) crystals. How crystals break, the structures afforded and their kinetics, are fundamental concepts that need to be understood in order to apply milling techniques during the formulation of tablets. This keynote lecture reviews some recent advances in the understanding of nucleation and milling processes in small molecule crystals.

First, I present our recent work in the study of the kinetics of nucleation of a series of four aromatic carboxylic acids in different solvents and at different supersaturations [1]. After computing a myriad of parameters, we found some revealing correlations between nucleation rates and the strength of the aromatic stacking involved in the growth of the crystals under study. This revealed, that the weakest continuous interaction, was controlling the kinetics of crystal nucleation at the molecular level.

Second, we study the kinetics of the conversion of theophylline into a solvate by milling. Theophylline converts into a 1:1 solvate and then into a 1:1.5 solvate with pyrrolidone upon grinding in increasing amounts of pyrrolidone. Whilst the kinetics of the conversion to the $1: 1$ solvate were very fast, formation of the 1:1.5 solvate was slow. We found that the kinetics of breaking the reactant crystals were controlling the kinetics of the overall process [2].

Third, we study a molecular system which can exist in two different polymorphs (A and B). Polymorph A can only be obtained by dry milling or milling in the presence of small amounts of solvent. Our investigations showed that our milling experiments always lead to the thermodynamically stable form. However, the thermodynamically stable form switches from B to A as the size of the crystallites becomes smaller [3].:

References:

[1] A.J. Cruz-Cabeza, R.J. Davey, S.S. Sachithananthan, R. Smith, S.K. Tang, T. Vetter, Y. Xiao, Chem Commun. 2017, 53, 7905-7908.

[2] D. Hasa, M. Pastore, M. Arhangelskis, B. Gabriele, A.J.

Cruz-Cabeza, G. Schneider-Rauber, A.D. Bond, and W. Jones, in submission, 2018.

[3] A. M. Belenguer, G. I. Lampronti, A. J. Cruz-Cabeza, C. A.

Hunter, and J. K. M. Sanders,

Chemical Science, 2016, 7, 6617.

Keywords: nucleation, milling, polymorphism 